STRUCTURAL BIOLOGY COMMUNICATIONS

ISSN 2053-230X

\section{Crystal structure of full-length Zika virus NS5 protein reveals a conformation similar to Japanese encephalitis virus NS5}

\author{
Anup K. Upadhyay,* Matthew Cyr, Kenton Longenecker, Rakesh Tripathi, \\ Chaohong Sun and Dale J. Kempf*
}

Received 23 December 2016

Accepted 31 January 2017

Edited by T. C. Terwilliger, Los Alamos National Laboratory, USA

Keywords: Zika virus; NS5; nonstructural protein 5 .

PDB reference: full-length Zika virus NS5 protein, $5 \mathrm{tfr}$

Supporting information: this article has supporting information at journals.iucr.org/f

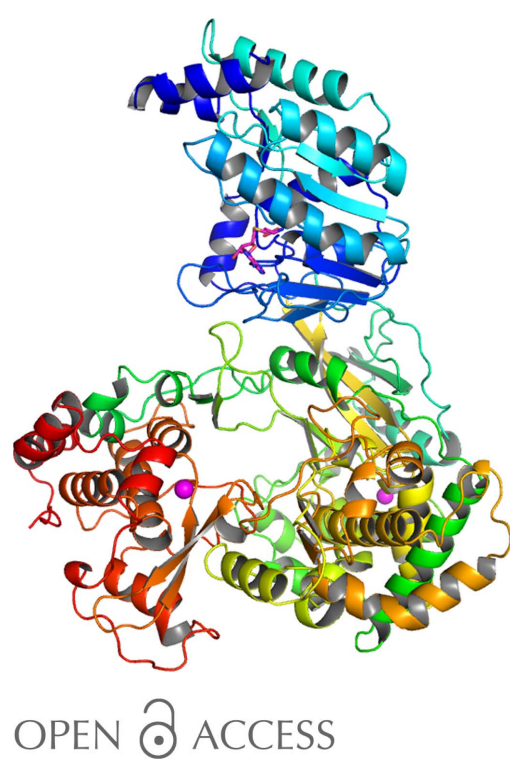

AbbVie Inc., 1 North Waukegan Road, North Chicago, IL 60064, USA. *Correspondence e-mail: anup.upadhyay@abbvie.com, dale.j.kempf@abbvie.com

The rapid spread of the recent Zika virus (ZIKV) epidemic across various countries in the American continent poses a major health hazard for the unborn fetuses of pregnant women. To date, there is no effective medical intervention. The nonstructural protein 5 of Zika virus (ZIKV-NS5) is critical for ZIKV replication through the $5^{\prime}$-RNA capping and RNA polymerase activities present in its N-terminal methyltransferase (MTase) and C-terminal RNA-dependent RNA polymerase (RdRp) domains, respectively. The crystal structure of the full-length ZIKV-NS5 protein has been determined at $3.05 \AA$ resolution from a crystal belonging to space group $P 2_{1} 2_{1} 2$ and containing two protein molecules in the asymmetric unit. The structure is similar to that reported for the NS5 protein from Japanese encephalitis virus and suggests opportunities for structure-based drug design targeting either its MTase or RdRp domain.

\section{Introduction}

The spread of Zika virus (ZIKV) through more than 35 countries in the American continents over the past year has created a major public health emergency (Lessler et al., 2016; Hajra et al., 2016). ZIKV is spread primarily through Aedes albopictus and $A$. aegypti mosquito vectors, but can also be sexually transmitted (Petersen et al., 2016; Weaver et al., 2016). Acute infection with ZIKV is often subclinical or, if symptomatic, typically associated with mild symptoms characteristic of acute viral infection. However, an increased risk of severe neurological disease, in particular Guillain-Barre syndrome, has been associated with ZIKV infection (Cao-Lormeau et al., 2016). Furthermore, ZIKV infection causes decreased male fertility in mice (Govero et al., 2016). Most concerning, however, is the microcephaly associated with viral replication in human fetal brain tissue following perinatal transmission in pregnant women (Garcez et al., 2016; Broutet et al., 2016; Carteaux et al., 2016).

$\mathrm{ZIKV}$ is a member of the Flaviviridae virus family, genus Flavivirus. It is closely related to other flaviviruses such as Dengue virus (DENV1-4), Yellow fever virus (YFV), Japanese encephalitis virus (JEV) and West Nile virus (WNV) (Petersen et al., 2016; Weaver et al., 2016). Flaviviridae are single positive-stranded RNA viruses that undergo cytoplasmic replication in host cells by a replication complex containing nonstructural protein 5 (NS5) as the RNA polymerase. Somewhat more distantly related are members of the genus Hepacivirus, which include the important human pathogen Hepatitis C virus (HCV; Giangaspero et al., 2008). Nucleoside 
and non-nucleoside inhibitor classes targeting the HCV NS5B RNA polymerase have been shown to be effective in combination with other inhibitor classes to profoundly block viral replication and cure HCV infection (Zhang et al., 2016).

Like other flaviviruses, ZIKV contains a linear genome encoding, from the $5^{\prime}$-end, three structural proteins (capsid, membrane and envelope) and seven nonstructural proteins (NS1, NS2a, NS2b, NS3, NS4a, NS4b and NS5) (Cunha et al., 2016). The NS5 proteins from flaviviruses are unique among RNA viruses for having a fused domain architecture comprised of an N-terminal RNA methyltransferase (MTase) domain and a C-terminal RNA-dependent RNA polymerase (RdRp) domain (Fig. 1a). The N-terminal MTase domain is responsible for $5^{\prime}$ capping and thereby stabilizes the viral RNA genome, while the C-terminal RdRp domain is critical for viral RNA replication (Decroly et al., 2011; Zhao, Soh, Lim et al., 2015; Lu \& Gong, 2013). Both of these two domains are potential therapeutic targets for developing antiviral drugs. X-ray crystal structures of the NS5 proteins from various flaviviruses have been solved and used for the structure-based drug design of novel antivirals (Lim et al., 2011; Benmansour et al., 2016; Malet et al., 2008). Here, we report the first X-ray crystal structure of the full-length NS5 polymerase from ZIKV in complex with $S$-adenosylhomocysteine (SAH), with the hope of aiding efforts towards the identification of antiviral drugs to help mitigate current and future ZIKV epidemics.

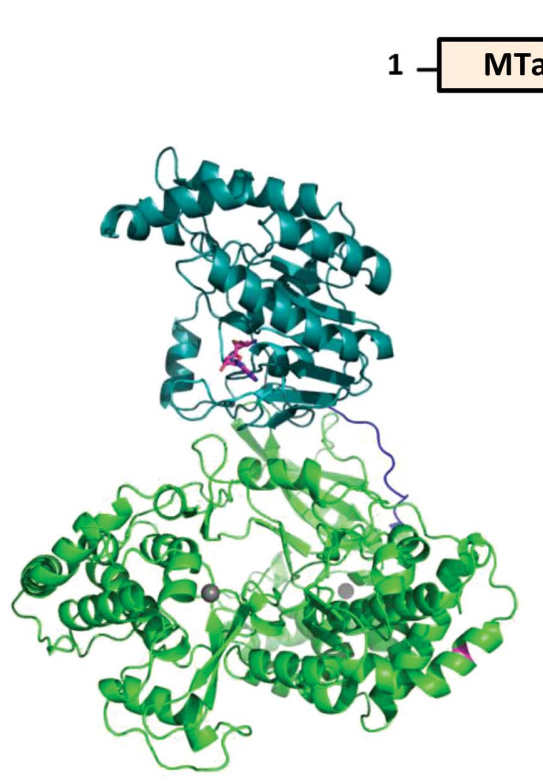

(b)

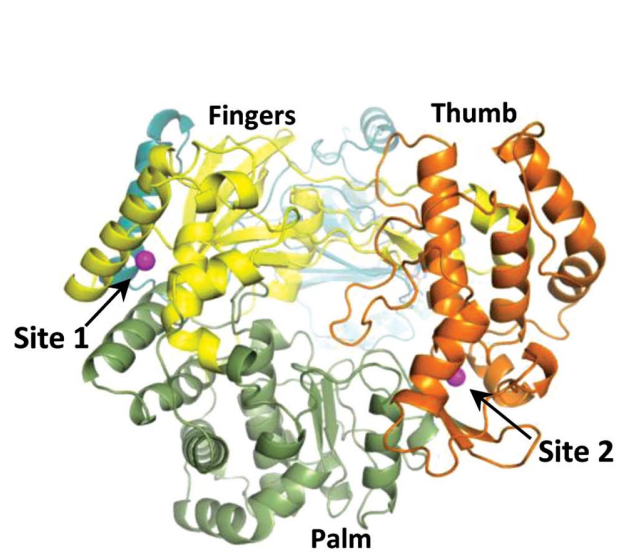

(e)

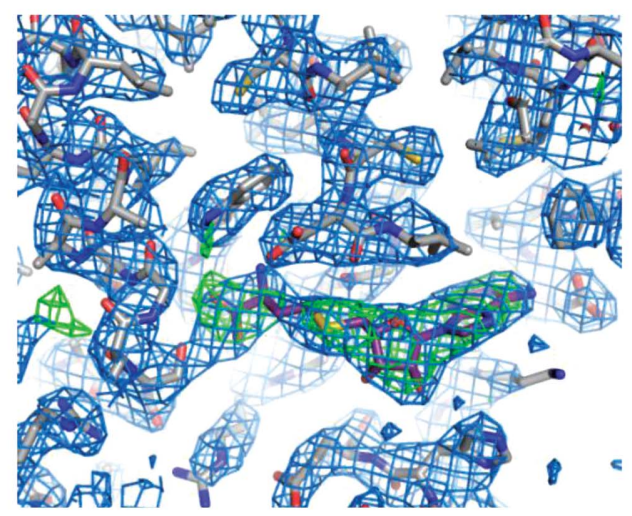

(c)

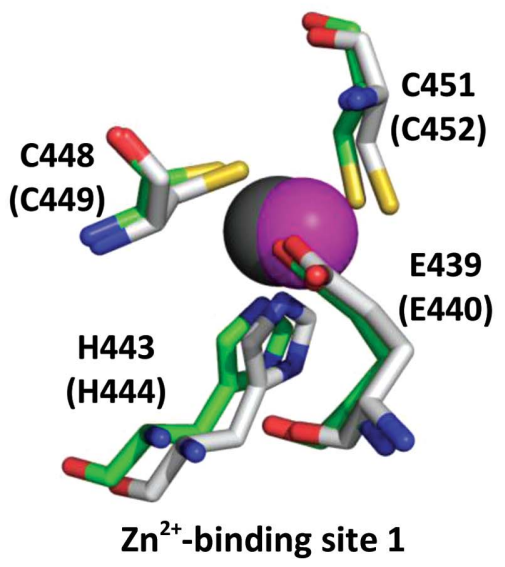

$(f)$

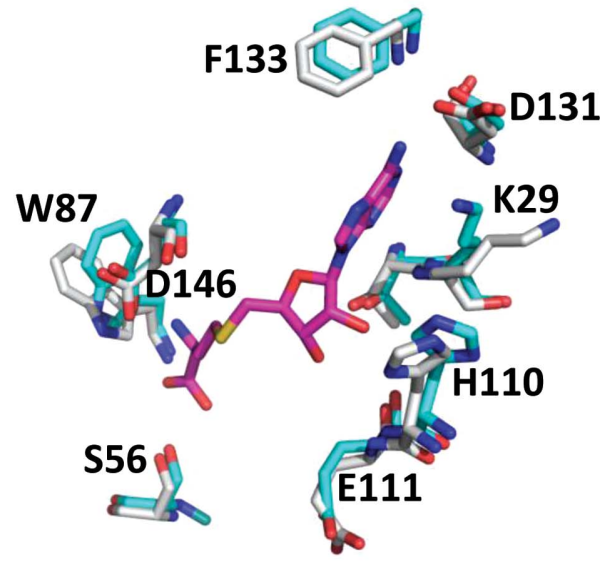

(d)

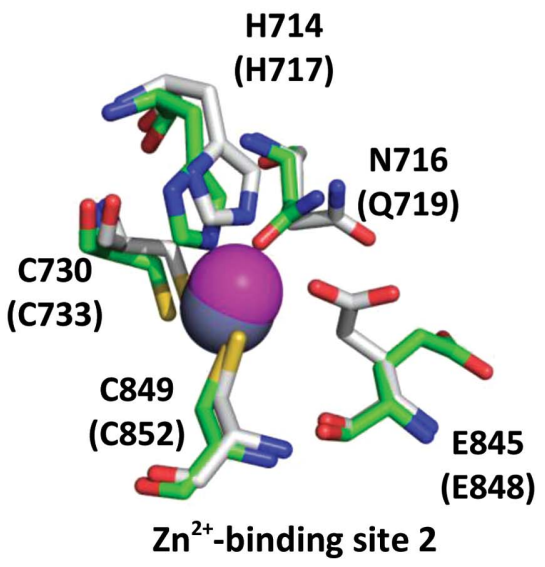

$(g)$

Figure 1

(a) Domain architecture of ZIKV-NS5(1-903) protein. (b) X-ray structure of the full-length ZIKV-NS5 protein, where residues 6-887 are observed in the structure. The MTase domain is shown in teal and the RdRp domain is shown in green. The bound cofactor (SAH) in the MTase domain is highlighted in magenta, and the two $\mathrm{Zn}^{2+}$ ions in the $\mathrm{RdRp}$ domain are shown in gray. The linker region connecting the MTase and $\mathrm{RdRp}$ domains is shown in blue. (c) Representative section of the electron-density map for the protein with the bound cofactor (SAH) in the MTase domain. The weighted $2 F_{\mathrm{o}}-F_{\mathrm{c}}$ map (blue) is contoured at $1 \sigma$ and a difference OMIT map (green) for the SAH is contoured at $3 \sigma .(d)$ The conserved residues involved in SAH (magenta) binding in the NS5 MTase domain are shown for the JEV-NS5 (gray; PDB entry 4k6m) and ZIKV-NS5 (cyan; PDB entry 5tfr) structures. (e) ZIKV-NS5 pol oriented with a 'right-handed' view of the palm (olive), thumb (orange) and fingers (yellow) domains, where the Mtase (blue) domain extends into the page. $(f, g)$ Coordination environments of the two $\mathrm{Zn}^{2+}$-binding sites. ZIKV-NS5 residues are shown in green and the two $\mathrm{Zn}^{2+}$ ions are shown in gray. JEV-NS5 residues are shown in gray and the corresponding $\mathrm{Zn}^{2+}$ ions are shown in magenta. 


\section{Materials and methods}

\subsection{Protein expression and purification}

The cDNA sequence encoding the full-length Zika virus strain MR766 RNA-dependent RNA polymerase NS5 protein ZIKV-NS5(1-903) (NCBI Reference Sequence YP_009227205.1) was synthetically generated (GenScript) and cloned into $\mathrm{pET}$-28b expression vector as a N-terminally $\mathrm{His}_{6}$ tagged protein with an internal thrombin cleavage site for removal of the $\mathrm{His}_{6}$ tag after purification. The recombinant protein was expressed in Escherichia coli BL21-CodonPlus (DE3)-RIPL strain. The cells were grown in Luria Broth (LB) medium containing $50 \mathrm{mg} \mathrm{l}^{-1}$ kanamycin and $34 \mathrm{mg} \mathrm{l}^{-1}$ chloramphenicol at $37^{\circ} \mathrm{C}$ until the cell density $\left(\mathrm{OD}_{600}\right)$ reached 0.4 ; the temperature was then lowered to $16^{\circ} \mathrm{C}$. Protein expression was induced by adding $0.5 \mathrm{~m} M$ IPTG to the medium and growing the cells overnight at $16^{\circ} \mathrm{C}$. The cell pellet was resuspended in lysis buffer $(20 \mathrm{~m} M$ Tris- $\mathrm{HCl} \mathrm{pH}$ 7.5, $500 \mathrm{~m} M \mathrm{NaCl}, 10 \mathrm{~m} M$ imidazole, $10 \%$ glycerol, $1 \mathrm{~m} M$ $\mathrm{MgCl}_{2}, 1 \mathrm{~m} M$ TCEP) containing EDTA-free proteaseinhibitor cocktail set V (Calbiochem, catalog No. 539137) and 50 units of turbonuclease (Accelagen, catalog No. N0103M) per gram of cell paste. The resuspended cells were lysed by passage through an emulsifier at $83 \mathrm{MPa}$ pressure. The cell lysate was clarified by centrifugation at $25000 \mathrm{~g}$ for $1 \mathrm{~h}$ and the supernatant was loaded onto a nickel-affinity column preequilibrated in the lysis buffer. The bound proteins were eluted by running a linear gradient of 10-500 $\mathrm{m} M$ imidazole over 20 column volumes and collected as $5 \mathrm{ml}$ fractions. The fractions containing the desired ZIKV-NS5(1-903) protein were pooled together and digested with thrombin (Sigma, catalog No. T4648) while dialyzing overnight at $4{ }^{\circ} \mathrm{C}$ in $20 \mathrm{mM}$ Tris- $\mathrm{HCl} \mathrm{pH} 7.5$ buffer containing $500 \mathrm{mM} \mathrm{NaCl}, 10 \%$ glycerol and $1 \mathrm{~m} M$ TCEP. The cleaved $\mathrm{His}_{6}$ tag and thrombin were removed by passing the thrombin-treated protein through $5 \mathrm{ml}$ Ni Sepharose 6 Fast Flow resin (GE Healthcare) and $1 \mathrm{ml} p$-aminobenzamidine-agarose beads (Sigma, catalog No. A-7155), respectively. The cleaved protein was further purified by size-exclusion chromatography on a Superdex S200 gel-filtration column using $20 \mathrm{~m} M$ Tris- $\mathrm{HCl}$ pH 7.5, $500 \mathrm{~m} M \mathrm{NaCl}, 10 \%$ glycerol, $1 \mathrm{~m} M$ TCEP as the final protein buffer. The purity of the purified protein was assessed by polyacrylamide gel electrophoresis (SDS-PAGE). The protein concentration was determined by UV absorbance at $280 \mathrm{~nm}$ with a NanoDrop 2000 spectrophotometer (Thermo Scientific) using a theoretical molar extinction coefficient of $224890 \mathrm{M}^{-1} \mathrm{~cm}^{-1}$.

\subsection{Crystallization}

The purified protein was concentrated to $12 \mathrm{mg} \mathrm{ml}^{-1}$ and set up for crystallization at $17^{\circ} \mathrm{C}$. The final optimized crystals were obtained from hanging-drop conditions using $0.1 \mathrm{M}$ sodium cacodylate $(\mathrm{pH} 5.9-6.9)$ and trisodium citrate (0.6$1.2 \mathrm{M}$ ) as well solutions. For data collection, the crystals were cryoprotected by dipping them into a solution containing $80 \%$ well solution and $20 \%(v / v)$ glycerol and then flash-cooled in liquid nitrogen. Crystallization information is given in Table 1.
Table 1

Crystallization conditions.

\begin{tabular}{ll}
\hline Method & Hanging-drop vapour diffusion \\
Plate type & 24-well plates \\
Temperature (K) & 290 \\
Protein concentration $\left(\mathrm{mg} \mathrm{ml}^{-1}\right)$ & 12 \\
Buffer composition of protein & $20 \mathrm{~m} M$ Tris- $\mathrm{HCl} \mathrm{pH} \mathrm{7.5,500} \mathrm{mM} \mathrm{NaCl,}$ \\
$\quad$ solution & $10 \%$ glycerol, $1 \mathrm{~m} M$ TCEP \\
Composition of reservoir solution & $0.1 M$ sodium cacodylate $(\mathrm{pH} 5.9-6.9)$, \\
& trisodium citrate $(0.6-1.2 M)$ \\
Volume and ratio of drop & $1: 1$ \\
Volume of reservoir $(\mathrm{ml})$ & 0.8 \\
\hline
\end{tabular}

Table 2

Data collection and processing.

Values in parentheses are for the outer shell.

\begin{tabular}{ll}
\hline Diffraction source & 17 -ID IMCA-CAT, APS \\
Wavelength $(\AA)$ & 1.00 \\
Temperature $(\mathrm{K})$ & 100 \\
Detector & Dectris PILATUS 6M \\
Crystal-to-detector distance $(\mathrm{mm})$ & 450 \\
Rotation range per image $\left({ }^{\circ}\right)$ & 0.3 \\
Total rotation range $\left({ }^{\circ}\right)$ & 180 \\
Exposure time per image $(\mathrm{s})$ & 0.1 \\
Space group & $P 2_{1} 2_{1} 2$ \\
$a, b, c(\AA)$ & $136,196,95$ \\
$\alpha, \beta, \gamma\left({ }^{\circ}\right)$ & $90,90,90$ \\
Mosaicity $\left({ }^{\circ}\right)$ & $0.1-0.2$ \\
Resolution range $(\AA)$ & $100-3.05(3.10-3.05)$ \\
Total No. of reflections & $330316(15565)$ \\
No. of unique reflections & $49315(2433)$ \\
Completeness $(\%)$ & $99.99(99.6)$ \\
Multiplicity & $6.7(6.4)$ \\
$\langle I / \sigma(I)\rangle$ & $8(2.1)$ \\
$R_{\text {meas }}$ & $23(99)$ \\
Overall $B$ factor from Wilson plot $\left(\AA^{2}\right)$ & 68 \\
\hline
\end{tabular}

\subsection{Data collection and processing}

Multiple crystals were screened to identify a crystal with reasonable diffraction. The best data set was collected to $3.05 \AA$ resolution under gaseous nitrogen $(100 \mathrm{~K})$ on the IMCA-CAT beamline (17-ID) at the Advanced Photon Source at Argonne National Laboratory, Argonne, Illinois, USA using X-rays at a wavelength of $1.0 \AA$. Diffraction intensities were processed using autoPROC (Vonrhein et al., 2011). Data-collection and refinement statistics are shown in Table 2.

\subsection{Structure solution and refinement}

The structure was solved by molecular replacement with the coordinates of JEV-NS5 (PDB entry 4k6m; Lu \& Gong, 2013) using Phaser within the CCP4 program suite (Winn et al., 2011; Table 3). The model was rebuilt using Coot (Emsley et al., 2010) and refined against structure factors using autoBUSTER (Smart et al., 2012). The two molecules in the asymmetric unit are very similar, with the protein structure built as a continuous chain for residues 6-887. Both exhibit additional densities for the bound cofactor $S$-adenosylhomocysteine (SAH) in each MTase domain and two zinc ions bound on each polymerase domain. With significant anisotropy in the diffraction data (anisotropic ratio $=1.3$ ), treatment with the StarAniso algorithm (Global Phasing Ltd) aided iterative refinement of 
Table 3

Structure solution and refinement.

Values in parentheses are for the outer shell.

\begin{tabular}{ll}
\hline Resolution range $(\AA)$ & $45-3.05(3.13-3.05)$ \\
Completeness $(\%)$ & $99.9(99.6)$ \\
No. of reflections, working set & $49224(3447)$ \\
No. of reflections, test set & $2471(154)$ \\
Final $R_{\text {cryst }}$ & $0.188(0.262)$ \\
Final $R_{\text {free }}$ & $0.237(0.353)$ \\
No. of non-H atoms & \\
$\quad$ Protein & 14188 \\
$\quad$ Ion & 4 \\
$\quad$ Ligand & 52 \\
$\quad$ Water & 229 \\
$\quad$ Total & 14473 \\
R.m.s. deviations & \\
$\quad$ Bonds $(\AA)$ & 0.010 \\
$\quad$ Angles $\left({ }^{\circ}\right)$ & 1.14 \\
Average $B$ factors $\left(\AA^{2}\right)$ & \\
$\quad$ Protein & 61 \\
$\quad$ Ion & 71 \\
$\quad$ Ligand & 70 \\
$\quad$ Water & 38 \\
Ramachandran plot & \\
$\quad$ Most favored $(\%)$ & 97.3 \\
Allowed $(\%)$ & 2.5 \\
\hline
\end{tabular}

the model. Figures were prepared using PyMOL (Schrödinger).

\section{Results}

Full-length Zika virus NS5 protein [ZIKV-NS5(1-903)] was purified from an E. coli-based expression system and the purified protein was crystallized at $17^{\circ} \mathrm{C}$. The structure was solved at $3.05 \AA$ resolution by molecular replacement using the published JEV-NS5 structure (PDB entry $4 \mathrm{k} 6 \mathrm{~m}$ ) as a search model (Table 3; Lu \& Gong, 2013). Residues 6-887 of the protein sequence were modeled as a continuous chain into the observed electron-density map (Fig. 1b). The N-terminal MTase domain showed additional density for the bound cofactor SAH that was copurified from the bacterial expression host (Fig. 1c). SAH is the demethylated form of $S$-adenosylmethionine (SAM), which is the major methyl donor for eukaryotic and prokaryotic methyltransferase enzymes. Co-purifications of SAH- or SAM-bound methyltransferase enzymes from bacterial expression hosts are quite common and have been reported previously for the DENV and ZIKV NS5 proteins (Coloma et al., 2016; Egloff et al., 2002; Lim et al., 2011). The residues surrounding the SAHbinding sites are conserved and adopt similar conformations among the related structures (Fig. 1d).

The C-terminal RdRp domain of the ZIKV-NS5 protein adopts the thumb, palm and fingers motifs (Fig. 1e) characteristic of polymerase folds, as reported for other flavivirus NS5 proteins and the distantly related HCV-NS5B protein ( $\mathrm{Lu}$ \& Gong, 2013; Sesmero \& Thorpe, 2015). There are two zincbinding sites in the RdRp domain. Site 1 (Fig. $1 f$ ) is conserved in JEV-NS5 and binds a $\mathrm{Zn}^{2+}$ ion by coordinating through residues Glu439, His443, Cys448 and Cys451. Site 2 (Fig. 1g) utilizes residues His714, Cys730 and Cys849 as in JEV, but orients the side chain of the fourth residue (Glu845) away and uses Asn716 instead of Gln as the fifth residue to coordinate the zinc. Site 2 has been suggested to play a regulatory role in modulating movement between the thumb and the palm of the RdRp domain (Lu \& Gong, 2013; Yap et al., 2007); however, the functional consequence of this subtle structural difference is not yet well understood.

The MTase domain of the ZIKV-NS5 protein folds onto the $\mathrm{RdRp}$ domain in a manner similar to that observed in the JEV-NS5 structure (Lu \& Gong, 2013). Unlike the JEV-NS5 structure, the linker region (residues 266-275) connecting the MTase and RdRp domains has continuous electron density to enable modeling of all of the residues. The relative spatial orientation of the two domains in the ZIKV-NS5 protein is similar ( $0.94 \AA$ r.m.s.d.) to that of the JEV-NS5 protein (Fig. 2a) but distinctly different from the DENV3-NS5 protein (Fig. 2b; PDB entry 5ccv; Klema et al., 2016). Consistent with the $67 \%$ sequence identity (Fig. $2 c$ ) and the observed structural similarities (Fig. $2 a$ ) between the ZIKV-NS5 and JEVNS5 proteins, the residues present at the interface of the two domains are highly conserved between these two proteins and also adopt similar conformations (Fig. $2 d$ ). The RdRp and MTase domains in flaviviral NS5 proteins are reported to cross-talk with each other to synergize their RNA polymerase and $5^{\prime}$-capping functions (Zhao, Soh, Chan et al., 2015; Zhao, Soh, Zheng et al., 2015). The linker region and the interacting residues at the interface of the two domains are shown to play critical roles in regulating the enzymatic functions of flaviviral NS5 proteins and impact viral replication (Zhao, Soh, Zheng et al., 2015). Compounds that destabilize this inter-domain interaction may therefore function as potential allosteric inhibitors for flaviviral NS5 enzymes.

\section{Discussion}

ZIKV is an example of several pathogenic human viruses that disproportionally affect people living in poverty with limited access to healthcare (Hotez et al., 2009). The expansion of Chikungunya virus and Dengue virus (Furuya-Kanamori et al., 2016) and the recent west African outbreak of Ebola virus (WHO Ebola Response Team, 2016) illustrate the need for cooperation between the public and private sectors to develop new antiviral drugs to aid in responding to future epidemics. The availability of detailed structural information from X-ray crystallographic studies on viral proteins can assist the discovery of new agents alongside high-throughput screening, repurposing of known antivirals and other drug-discovery techniques (Eyer et al., 2016; Elfiky, 2016; Julander et al., 2016). In particular, iterative structure-based drug design based on co-crystallography of enzyme-inhibitor structures of viral polymerases has been especially fruitful in the optimization of agents for other viral diseases (Lim et al., 2011; Sesmero \& Thorpe, 2015). Here, we report the first X-ray crystal structure of the full-length NS5 protein from ZIKV, containing both MTase and RdRp domains. We anticipate that this structure may enable the discovery and structure-based 
optimization of new agents to treat or possibly prevent ZIKV infection.

The current ZIKV-NS5 structure compares very closely with the published JEV-NS5 structure (Fig. 2a), suggesting a similar behavior in functional properties and inter-domain dynamics. While the domain positioning of ZIKV-NS5 contrasts with those reported for DENV-NS5 structures, the individual domains in these proteins share similar structural features, as expected from the high sequence homology among the flaviviruses. The diffraction data in the current study offer insight into the larger structural features, but the interpretation of detailed atomic positions remains limited. Future

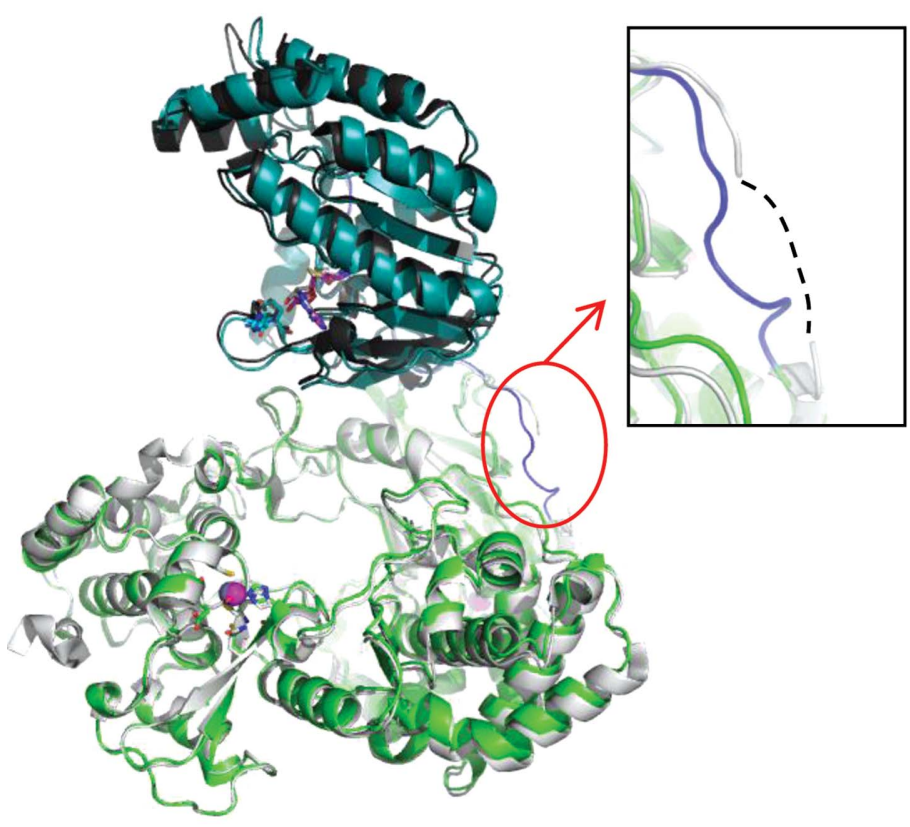

$(a)$

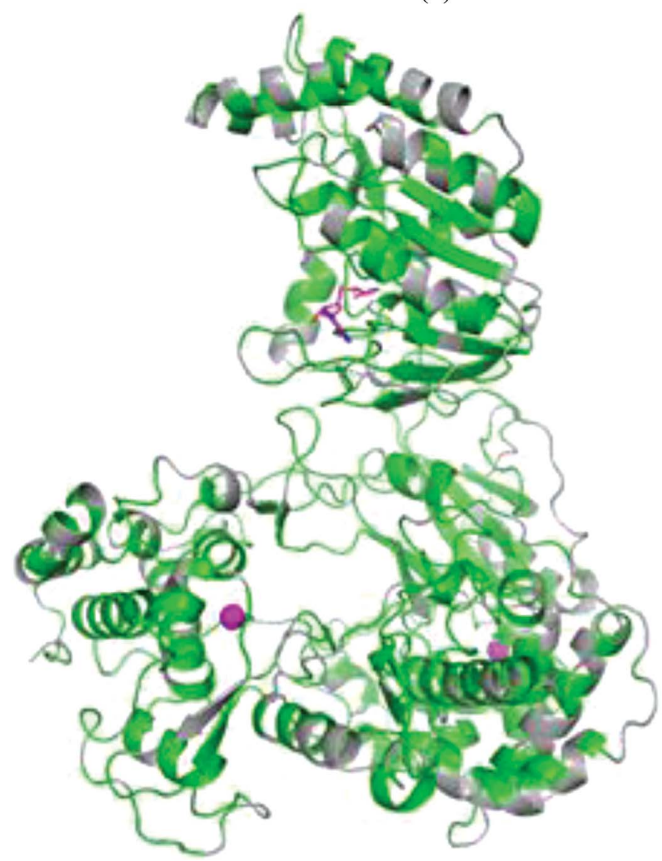

(c)

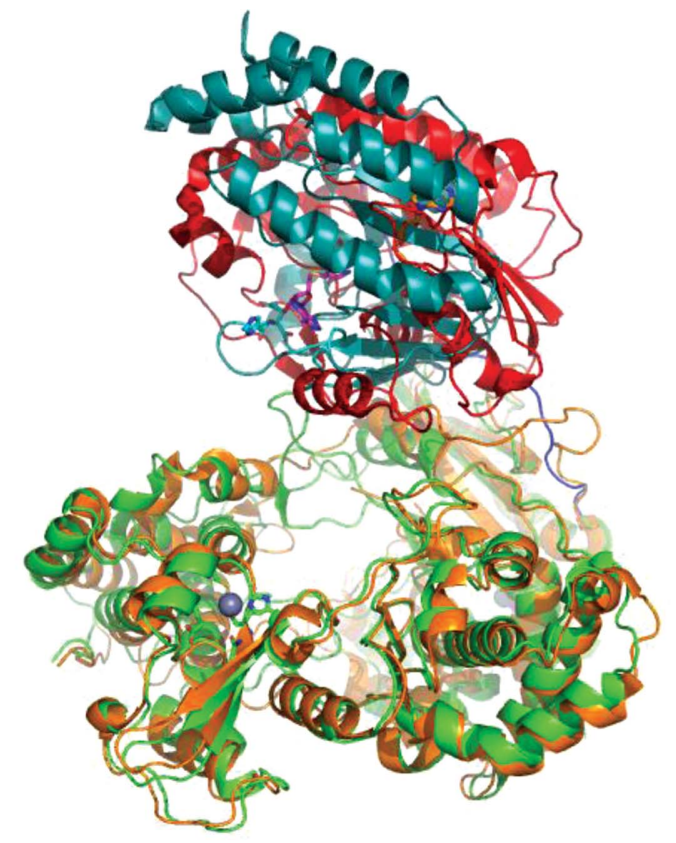

$(b)$

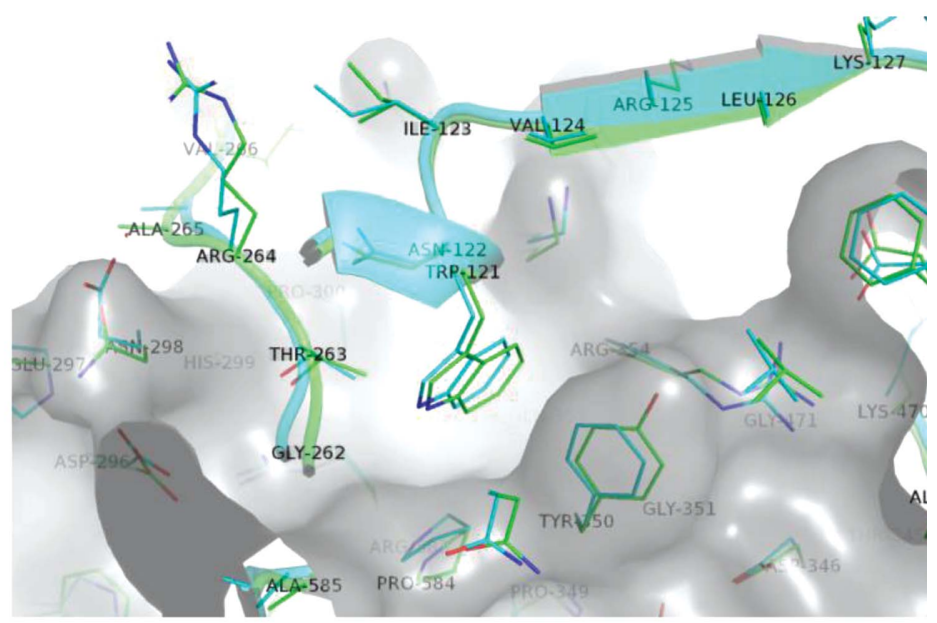

$(d)$

Figure 2

Comparison of NS5 structures from ZIKV (PDB entry 5tfr), JEV (PDB entry 4k6m) and DENV3 (PDB entry 5ccv). (a) Overlay of the full-length ZIKVNS5 structure with the JEV-NS5 structure (PDB entry 4k6m). The JEV-NS5 protein is shown in gray. The ZIKV-NS5 protein is shown in teal (MTase domain) and green (RdRp domain). The inset shows the linker regions of the two proteins (blue, ZIKV-NS5; gray, JEV-NS5). (b) Overlay of the DENV3-NS5 structure with the ZIKV-NS5 protein. The DENV3-NS5 protein is shown in red (MTase domain) and orange (RdRp domain). The ZIKVNS5 protein is shown as in $(a)$. (c) Sequence homology between the ZIKV-NS5 and JEV-NS5 proteins is shown by highlighting the conserved residues between the two proteins in green on the ZIKV-NS5 structure. (d) Structural overlay of the ZIKV-NS5 and JEV-NS5 proteins at the interface between the MTase and RdRp domains with conserved residues involved in the interaction. 
studies should allow structural characterization at higher resolution with more details of local features. Some strategies that are likely to improve diffraction quality include truncations of the currently disordered $\mathrm{N}$-terminal (amino acids $1-5$ ) and C-terminal (amino acids 888-903) residues of the protein to promote different crystal packing. This may also reduce the anisotropic diffraction pattern that we have observed in the current crystal form (see \$2.4). Alternatively, crystallization of the individual MTase and RdRp domains, as evident from the recently published high-resolution X-ray structure of the ZIKV-NS5 MTase domain (Coloma et al., 2016), may also aid in obtaining high-resolution structural information on the individual domains. In addition, further characterizations through biophysical and biochemical functional assays are needed to understand the structure-function relationships in the context of inhibitor screening.

In conclusion, the global reach of the ZIKV epidemic emphasizes the need for multiple approaches to stem its expansion and prevent its devastating sequelae in unborn children. Although the development of effective vaccines may have the greatest impact, new antivirals that block ZIKV replication may also be important for treating or preventing ZIKV-associated morbidities. Here, we report the first X-ray crystal structure of the full-length NS5 protein from ZIKV, which may be useful in the discovery and optimization of such antivirals.

\section{Acknowledgements}

AKU, KL, RT, CS and DJK are employees of AbbVie. This study was partially sponsored by AbbVie. MC performed his portion of this study at AbbVie as a Franciscan Institute for World Health (FIWH) Summer Intern in partnership with the AbbVie Neglected Diseases Program. AKU, CS and DJK designed the study. RT designed the expression constructs. $\mathrm{AKU}$ and $\mathrm{MC}$ performed the protein expression, purification, biophysical characterization and crystallization. KL performed the X-ray data collection, processing and structure determination. AKU, KL and DJK wrote the manuscript. All authors provided edits to the manuscript and reviewed the final version. Dr Jeffrey Rohde (Franciscan University, Steubenville, Ohio, USA) is acknowledged for his leadership and coordination of the FIWH intern program. William and Susan Reighard are gratefully acknowledged for financial support of FIWH. Use of the IMCA-CAT beamline 17-ID at the Advanced Photon Source was supported by the companies of the Industrial Macromolecular Crystallography Association through a contract with Hauptman-Woodward Medical Research Institute. This research used resources of the Advanced Photon Source, a US Department of Energy (DOE) Office of Science User Facility operated for the DOE Office of Science by Argonne National Laboratory under Contract No. DE-AC02-06CH11357.

\section{References}

Benmansour, F., Trist, I., Coutard, B., Decroly, E., Querat, G., Brancale, A. \& Barral, K. (2017). Eur. J. Med. Chem. 125, 865-880.
Broutet, N., Krauer, F., Riesen, M., Khalakdina, A., Almiron, M., Aldighieri, S., Espinal, M., Low, N. \& Dye, C. (2016). N. Engl. J. Med. 374, 1506-1509.

Cao-Lormeau, V. M. et al. (2016). Lancet, 387, 1531-1539.

Carteaux, G., Maquart, M., Bedet, A., Contou, D., Brugières, P., Fourati, S., Cleret de Langavant, L., de Broucker, T., Brun-Buisson, C., Leparc-Goffart, I. \& Mekontso Dessap, A. (2016). N. Engl. J. Med. 374, 1595-1596.

Coloma, J., Jain, R., Rajashankar, K. R., García-Sastre, A. \& Aggarwal, A. K. (2016). Cell. Rep. 16, 3097-3102.

Cunha, M. S. et al. (2016). Genome Announc. 4, e00032-16.

Decroly, E., Ferron, F., Lescar, J. \& Canard, B. (2011). Nature Rev. Microbiol. 10, 51-65.

Egloff, M.-P., Benarroch, D., Selisko, B., Romette, J.-L. \& Canard, B. (2002). EMBO J. 21, 2757-2768.

Elfiky, A. A. (2016). J. Med. Virol. 88, 2044-2051.

Emsley, P., Lohkamp, B., Scott, W. G. \& Cowtan, K. (2010). Acta Cryst. D66, 486-501.

Eyer, L., Nencka, R., Huvarová, I., Palus, M., Alves, M. J., Gould, E. A., De Clercq, E. \& Růžek, D. (2016). J. Infect. Dis. 214, 707-711.

Furuya-Kanamori, L., Liang, S., Milinovich, G., Soares Magalhaes, R. J., Clements, A. C., Hu, W., Brasil, P., Frentiu, F. D., Dunning, R. \& Yakob, L. (2016). BMC Infect. Dis. 16, 84.

Garcez, P. P., Loiola, E. C., Madeiro da Costa, R., Higa, L. M., Trindade, P., Delvecchio, R., Nascimento, J. M., Brindeiro, R., Tanuri, A. \& Rehen, S. K. (2016). Science, 352, 816-818.

Giangaspero, M., Harasawa, R. \& Zanetti, A. (2008). J. Virol. Methods, 153, 280-299.

Govero, J., Esakky, P., Scheaffer, S. M., Fernandez, E., Drury, A., Platt, D. J., Gorman, M. J., Richner, J. M., Caine, E. A., Salazar, V., Moley, K. H. \& Diamond, M. S. (2016). Nature (London), 540, 438-442.

Hajra, A., Bandyopadhyay, D. \& Hajra, S. K. (2016). N. Am. J. Med. Sci. 8, 123-128.

Hotez, P. J., Fenwick, A., Savioli, L. \& Molyneux, D. H. (2009). Lancet, 373, 1570-1575.

Julander, J. G., Siddharthan, V., Evans, J., Taylor, R., Tolbert, K., Apuli, C., Stewart, J., Collins, P., Gebre, M., Neilson, S., Van Wettere, A., Lee, Y.-M., Sheridan, W. P., Morrey, J. D. \& Babu, Y. S. (2017). Antiviral Res. 137, 14-22.

Klema, V. J., Ye, M., Hindupur, A., Teramoto, T., Gottipati, K., Padmanabhan, R. \& Choi, K. H. (2016). PLoS Pathog. 12, e1005451.

Lessler, J., Chaisson, L. H., Kucirka, L. M., Bi, Q., Grantz, K., Salje, H., Carcelen, A. C., Ott, C. T., Sheffield, J. S., Ferguson, N. M., Cummings, D. A. T., Metcalf, C. J. \& Rodriguez-Barraquer, I. (2016). Science, 353, aaf8160.

Lim, S. P. et al. (2011). J. Biol. Chem. 286, 6233-6240.

Lu, G. \& Gong, P. (2013). PLoS Pathog. 9, e1003549.

Malet, H., Massé, N., Selisko, B., Romette, J.-L., Alvarez, K., Guillemot, J. C., Tolou, H., Yap, T. L., Vasudevan, S., Lescar, J. \& Canard, B. (2008). Antiviral Res. 80, 23-35.

Petersen, L. R., Jamieson, D. J., Powers, A. M. \& Honein, M. A. (2016). N. Engl. J. Med. 374, 1552-1563.

Sesmero, E. \& Thorpe, I. F. (2015). Viruses, 7, 3974-3994.

Smart, O. S., Womack, T. O., Flensburg, C., Keller, P., Paciorek, W., Sharff, A., Vonrhein, C. \& Bricogne, G. (2012). Acta Cryst. D68, 368-380.

Vonrhein, C., Flensburg, C., Keller, P., Sharff, A., Smart, O., Paciorek, W., Womack, T. \& Bricogne, G. (2011). Acta Cryst. D67, $293-$ 302.

Weaver, S. C., Costa, F., Garcia-Blanco, M. A., Ko, A. I., Ribeiro, G. S., Saade, G., Shi, P.-Y. \& Vasilakis, N. (2016). Antiviral Res. 130, 69-80.

WHO Ebola Response Team (2016). N. Engl. J. Med. 375, 587-596. Winn, M. D. et al. (2011). Acta Cryst. D67, 235-242.

Yap, T. L., Xu, T., Chen, Y.-L., Malet, H., Egloff, M.-P., Canard, B., Vasudevan, S. G. \& Lescar, J. (2007). J. Virol. 81, 4753-4765. 
Zhang, J., Nguyen, D. \& Hu, K.-Q. (2016). N. Am. J. Med. Sci. 9, 47-54.

Zhao, Y., Soh, T. S., Chan, K. W. K., Fung, S. S. Y., Swaminathan, K., Lim, S. P., Shi, P.-Y., Huber, T., Lescar, J., Luo, D. \& Vasudevan, S. G. (2015). J. Virol. 89, 10717-10721.

Zhao, Y., Soh, T. S., Lim, S. P., Chung, K. Y., Swaminathan, K.,
Vasudevan, S. G., Shi, P.-Y., Lescar, J. \& Luo, D. (2015). Proc. Natl Acad. Sci. USA, 112, 14834-14839.

Zhao, Y., Soh, T. S., Zheng, J., Chan, K. W. K., Phoo, W. W., Lee, C. C., Tay, M. Y. F., Swaminathan, K., Cornvik, T. C., Lim, S. P., Shi, P.-Y., Lescar, J., Vasudevan, S. G. \& Luo, D. (2015). PLoS Pathog. 11, e1004682. 